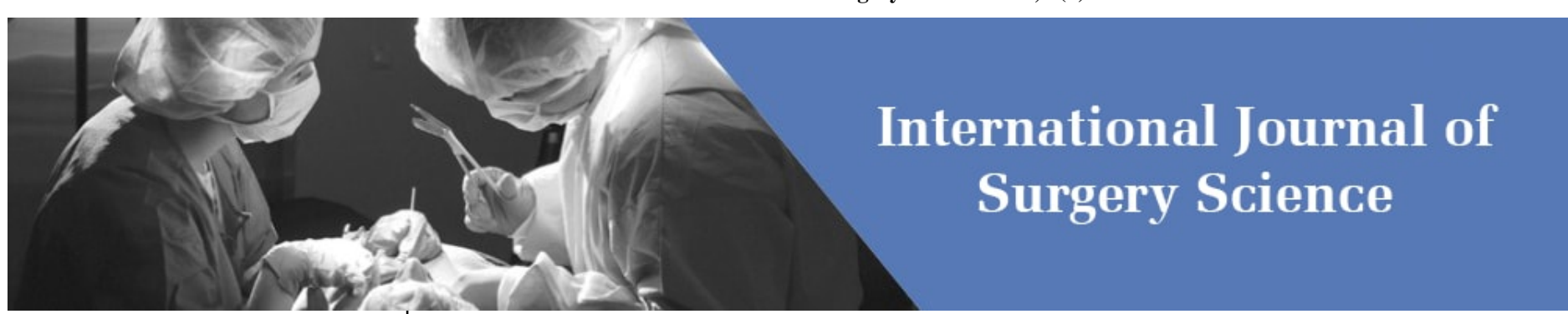

E-ISSN: 2616-3470

P-ISSN: 2616-3462

(C) Surgery Science

www.surgeryscience.com

2021; 5(4): 23-25

Received: 16-08-2021

Accepted: 25-09-2021

\section{Ahmed Elshiekh}

University Hospitals Coventry and Warwickshire NHS Trust, United

Kingdom

Ahmed Mohammad Hassan

University Hospitals Coventry and

Warwickshire NHS Trust, United

Kingdom

\section{Faisal Kahloon}

University Hospitals Coventry and

Warwickshire NHS Trust, United

Kingdom

\section{Asif Mahmood}

University Hospitals Coventry and Warwickshire NHS Trust, United Kingdom
Corresponding Author: Ahmed Elshiekh

University Hospitals Coventry and Warwickshire NHS Trust, United Kingdom

\section{Diagnostic resources management before lower limb revascularization in a UK tertiary vascular unit, the guidelines versus the practice}

\author{
Ahmed Elshiekh, Ahmed Mohammad Hassan, Faisal Kahloon and Asif \\ Mahmood
}

DOI: https://doi.org/10.33545/surgery.2021.v5.i4a.756

\begin{abstract}
Background: Peripheral Arterial Disease (PAD) causes significant health and economic burden.
\end{abstract}

Nice guidelines recommend performing Doppler US (DUS) as the first line in all patients with PAD for whom re-vascularization is being considered. MRA should be performed if further imaging is needed before re-vascularization and CT angiography (CTA) is indicated only if MRA is not tolerated or contraindicated.

Some published studies showed superiority of contrast enhanced MRA over conventional diagnostic angiography.

On the basis of these guidelines and the published literature diagnostic invasive lower limb angiography should not be routine practice because of the risks and the costs implications.

We performed a study to identify the adherence in our unit to these guidelines and the resulting impact on patient safety and service provision costs.

Methods: Retrospective data collection in a single large UK tertiary referral centre. Fifty-eight patients who underwent lower limb bypass surgery were included over a time period from June 2015 to June 2016.

Results: $75 \%$ of the patients (N: 44) had DUS as a first investigation, $29 \%$ (N: 17) had CTA or MRA as a second investigation and 39\% (N: 23) of the patients had diagnostic angiography preoperatively with an overall complication rate (diagnostic and therapeutic angiography) of $12 \%$.

The estimated costs of the angiographies during the one-year period were $£ 28,934$ ( $£ 1,258$ per patient).

Conclusion: Significant percentage of the patients going for lower limb bypass surgery are subject to unnecessary risk due to pre-operative diagnostic angiography with an overall increase in service costs. This is unjustified in the view of the current National guidelines and published literature.

Keywords: Tertiary vascular unit, Peripheral Arterial Disease (PAD), UK

\section{Introduction}

Peripheral Arterial Disease (PAD) causes significant health and economic burden. It is estimated that $20 \%$ of people aged over 60 years have some degree of peripheral arterial disease ${ }^{[1]}$.

Diagnostic modalities commonly used vary from simple non-invasive modalities like Doppler ultrasound (DUS), CT angiography (CTA), Magnetic resonance angiography (MRA) up to invasive procedures as diagnostic angiography.

Although invasive diagnostic angiography has been historically considered as the gold standard for diagnosis of PAD, simple non invasive procedures have been too shown to have very high sensitivity and specificity. Simple Doppler ultrasound (DUS) was shown to have $78 \%$ sensitivity and 99\% specificity for the diagnosis of popliteal artery PAD with even better results in femoral artery with a $100 \%$ specificity and $95 \%$ sensitivity ${ }^{[2]}$. CTA was shown to have high sensitivity (96\%) and specificity (95\%) ${ }^{[3]}$. MRA had a sensitivity of $93 \%$ and specificity of $94 \%$ and was even shown in more than one study to be superior to invasive angiography ${ }^{[4,5]}$.

Nice guidelines recommend performing Doppler US (DUS) as the first line in all the patients with PAD for whom re-vascularization is being considered. MRA should be performed if further imaging is needed before re-vascularization and CT angiography (CTA) is indicated only if MRA is not tolerated or contraindicated ${ }^{[6]}$. On the basis of these guidelines as well as the aforementioned literature diagnostic invasive lower limb angiography should not be the routine practice because of the risks and the costs implications. 
We performed a study to identify the adherence in our unit which is a big university hospital and tertiary referral centre in the UK to these guidelines and the resulting impact on patient safety and service provision costs.

\section{Patients \& Methods}

Retrospective data collection from the patient's records in a single large UK tertiary referral centre (University Hospital Coventry and Warwickshire) over a one-year time period from June 2015 to June 2016. All Patients who underwent lower limb bypass surgery were included except those who had an extraanatomical bypass.

Hospital research and development department approval was acquired.

\section{Results}

Fifty-eight patients who underwent lower limb bypass surgery from June 2015 to June 2016 were included.

$75 \%$ of these patients (N: 44) had DUS as a first investigation, $29 \%$ (N: 17) had CTA or MRA as a second investigation.

Of the patients included in our study $84 \%$ underwent angiography with $46 \%$ of them having diagnostic angiography and only 34\% having therapeutic angiography (N:49, N diagnostic:23, $\mathrm{N}$ therapeutic:17). Overall $39 \%$ of all patients included in our study had diagnostic angiography (N: 23/58).

Table one shows the numbers of patients who underwent each investigation and the order of the investigation in relation to other investigations done for the same patients.

Table 1: The numbers of patients who underwent each investigation and the order of the investigation in relation to other investigations done for the same patients.

\begin{tabular}{|c|c|c|c|c|}
\hline INV Order & US & MRI & CT & Angiography \\
\hline $1^{\text {st }}$ & $44(75 \%)$ & $5(8.6 \%)$ & $7(12 \%)$ & $2(3 \%)$ \\
\hline $2^{\text {nd }}$ & $2(3.8 \%)$ & $10(19 \%)$ & $7(13 \%)$ & $33(63 \%)$ \\
\hline $3^{\text {rd }}$ & $4(22 \%)$ & 0 & 0 & $14(77 \%)$ \\
\hline
\end{tabular}

The complications rate of angiography (diagnostic and therapeutic angiography) for patients included in our study was $12 \%$. The complications that occurred during our study included contrast leak, puncture site haematoma, dissection, retroperitoneal haemorrhage, blood transfusion and inability to tolerate the procedure. Table two shows the complications.

Table 1: The complications (* complications happening in the same patient)

\begin{tabular}{|c|c|c|c|c|}
\hline $\begin{array}{c}\text { Contrast } \\
\text { leak }\end{array}$ & Haematoma & $\begin{array}{c}\text { Procedure not } \\
\text { tolearted }\end{array}$ & $\begin{array}{c}\text { Arterial perforation, subintimal } \\
\text { dissection }\end{array}$ & $\begin{array}{c}\text { Retroperitoneal Haematoma, hypotension and } \\
\text { blood transfusion }\end{array}$ \\
\hline 1 & 2 & 1 & $1^{*}$ & $1^{*}$ \\
\hline
\end{tabular}

The estimated costs of the angiographies during the one-year period were $£ 28,934$ ( $£ 1,258$ per patient).

\section{Discussion}

Although lower limb angiography is still being though of as the gold standard for preoperative evaluation for lower limb ischaemia patients, less invasive modalities are not inferior and maybe even superior to it without carrying the same risks inherent to its nature as an invasive procedure. Lower limb angiography complications may be secondary to the vascular access (e.g. bleeding, infection, atheroembolism, dissection, vessel disruption or perforation, hematoma, pseudoaneurysm, arteriovenous fistula) or the utilization of contrast material. The complications rate in our study were $12 \%$. This was for both diagnostic and therapeutic procedures. In literature the complications rate for diagnostic angiography alone was lower than this at 1.9 to $2.9 \%{ }^{[7]}$. This maybe due to the fact that we did not include large number of patients.

The average costs of angiography ranges from $£ 1,135$ to $£ 1,995$ according to the data published in 2016/2017 by NHS England with costs of DUS, CTA and MRA being $£ 56, £ 120$ and $£ 211$ respectively(8). The costs of the diagnostic angiographies in our unit over a one-year period were estimated to be $£ 28,934$. However, the real cost was higher because it did not include the costs of the medical staff hours and the day case beds costs. Furthermore, if a complication happens the patient will need to be admitted to the hospital with a rise in overall cost. Thus the real cost saving that can be done by avoiding diagnostic angiographies would be much higher than this figure.

Overusing diagnostic tests is well reported in medicine. Examples include routine pre-operative chest X-rays, DEXA scans for suspected osteoporosis in women less than sixty years of age, CT scans for suspected pulmonary embolism in patients with a low probability score and others(9). Using invasive angiography that could be easily replaced by a non-invasive diagnostic test is another form of over-investigating in medicine.

\section{Conclusion}

Significant percentage of the patients going for lower limb bypass surgery are subject to unnecessary risk due to preoperative diagnostic angiography with an overall increase in service costs. This is unjustified in the view of the current National guidelines and published literature.

\section{References}

1. Marrett E, DiBonaventura M, Zhang Q. Burden of peripheral arterial disease in Europe and the United States: a patient survey. Health Qual Life Outcomes [Internet]. 2013 [cited 2017;11(1):175. Available from: http://hqlo.biomedcentral.com/articles/10.1186/1477-752511-175

2. Moneta GL, Yeager RA, Lee RW, Porter JM. Noninvasive localization of arterial occlusive disease: a comparison of segmental Doppler pressures and arterial duplex mapping. J Vasc Surg [Internet] 1993;17(3):578-82. Available from: http://www.ncbi.nlm.nih.gov/pubmed/8445755

3. Jens S, Koelemay MJW, Reekers JA, Bipat S. Diagnostic performance of computed tomography angiography and contrast-enhanced magnetic resonance angiography in patients with critical limb ischaemia and intermittent claudication: systematic review and meta-analysis. Eur Radiol [Internet]. 2013;23(11):3104-14. Available from: http://www.ncbi.nlm.nih.gov/pubmed/23801421

4. Leiner T, Kessels AGH, Schurink GW, Kitslaar PJEHM, de Haan MW, Tordoir JHM, et al. Comparison of contrastenhanced magnetic resonance angiography and digital subtraction angiography in patients with chronic critical ischemia and tissue loss. Invest Radiol [Internet]. 2004;39(7):435-44. Available from: http://www.ncbi.nlm.nih.gov/pubmed/15194915

5. Dorweiler B, Neufang A, Kreitner K-F, Schmiedt W, Oelert $\mathrm{H}$. Magnetic resonance angiography unmasks reliable target vessels for pedal bypass grafting in patients with diabetes 
mellitus. J Vasc Surg [Internet] 2002;35(4):766-72.

Available from: http://www.sciencedirect.com/science/article/pii/S07415214 02383393

6. Peripheral arterial disease: diagnosis and management| Guidance and guidelines | NICE. [cited 2017 Apr 9]; Available from: https://www.nice.org.uk/guidance/cg147

7. Balduf LM, Langsfeld M, Marek JM, Tullis MJ, Kasirajan K, Matteson B. Complication Rates of Diagnostic Angiography Performed by Vascular Surgeons. Vasc Endovascular Surg [Internet] 2002;36(6):439-45. Available from: http://www.ncbi.nlm.nih.gov/pubmed/12476233

8. NHS England» National Tariff Payment System 2016/17 [Internet]. [Cited 2017 Apr 9]. Available from: https://www.england.nhs.uk/resources/pay-syst/tariffconsultation-notice/

9. Rao VM, Levin DC. The Overuse of Diagnostic Imaging and the Choosing Wisely Initiative. Ann Intern Med [Internet]. 2012;157(8):574. Available from: http://annals.org/article.aspx?doi=10.7326/0003-4819-157-8201210160-00535 